\title{
Ecological Welfare of China's Forest Towns: Concept, Formation Mechanism, and Evaluation Index System
}

\author{
Chen Chen, ${ }^{1,2}$ Haitao Sun, ${ }^{2}$ and Yingli Huang $\mathbb{D}^{1}$ \\ ${ }^{1}$ School of Economics and Management, Northeast Forestry University, Harbin, 150040, China \\ ${ }^{2}$ College of Accounting and Finance, Heilongjiang Polytechnic, Harbin, 150010, China \\ Correspondence should be addressed to Yingli Huang; lqq2015010092@nefu.edu.cn
}

Received 15 January 2022; Revised 29 January 2022; Accepted 3 February 2022; Published 24 February 2022

Academic Editor: Thippa Reddy G

Copyright (c) 2022 Chen Chen et al. This is an open access article distributed under the Creative Commons Attribution License, which permits unrestricted use, distribution, and reproduction in any medium, provided the original work is properly cited.

The economic subsystem, social subsystem, and natural ecosystem of forest towns constitute forest towns' complex ecosystem. Forest towns' complex system provides good ecological welfare for the residents' survival and development, especially the beautiful ecological environment has played a positive role in promoting the residents' healthcare. First of all, this paper expounds on the concept of forest towns' ecological welfare from the construction goals of forest towns. Secondly, it analyzes the formation mechanism of forest towns' ecological welfare based on the DPSIR conceptual model. Finally, taking forest towns in Heilongjiang Province as an example, the index system for ecological welfare evaluation is constructed, and the influence degree of the evaluation index is analyzed through using the analytic hierarchy process. The results show that employment and income are the most important indicators that affect the ecological welfare of forest towns in Heilongjiang Province. This paper provides a theoretical basis for evaluating the level of ecological welfare level, level of healthcare level, and planning development path of Chinese forest towns in the future.

\section{Introduction}

Forest town is a town that relies on forest resources to provide ecological products and ecological services such as forest sightseeing, leisure vacation, sports, and health as its main characteristics. And it is a type of characteristic town that integrates industry, culture, tourism, and community functions. Since 2015, Zhejiang Province has taken the lead in the construction of forest towns. As of December 2020, there have been more than 1,000 provincial forest towns, and national-level forest characteristic towns also announced 50 construction pilot projects in August 2018. Forest towns' construction has set off an upsurge in China. The construction process of the forest town is a process of human-earth interaction. The natural ecosystem has the provisioning function with food and water supply, the regulation function with maintaining air quality and regulating the climate, the supporting function with producing oxygen and forming soil, and the cultural function with providing aesthetic appreciation, entertainment, and recreation for human beings [1]. It is an essential contribution to human survival and development and maintaining a good quality of life, including healthcare [2]. Human activities act on the natural ecosystem of the forest town, forming a "human and Earth circle" of deep penetration and interactive influence between nature, economy, and society. Forest towns develop a series of related industries such as accommodation, catering, and local specialties through tourism. It has brought into play the economic, ecological, and social benefits and maintained the healthcare of the residents of the forest town. However, due to the different development models of forest towns, the structure of the human-earth system is affected, and the ecological welfare of people is affected. In some forest towns, due to the limitations' technology and the management policies, the multiple functions of forest towns have not been fully utilized compared with the abundant forest resources, and even the forest ecological curse effect has appeared. Some forest towns adopt the extensive development model of "high investment, high consumption, and high emission." 
Although they have attracted more tourists and achieved economic and social development, the forest ecological environment faces severe pressure. The deterioration of the environment results in people's increasing demand about the ecological welfare which cannot be met. At present, the research on forest towns is in its infancy stage. The qualitative research conducted by most scholars on forest towns mainly focuses on the concept, characteristics, construction significance, and development path of forest towns [3-5]. And some scholars also discussed the distribution, characteristics, and influencing factors of forest towns from a spatial perspective [6]. Although some scholars have evaluated characteristic towns from different perspectives, the evaluation of forest towns, especially the study of ecological welfare evaluation, has not yet been formed [7-10]. In the construction of a "people-centered" town, bringing more ecological welfare to people is the ultimate goal of town construction and development.

Therefore, this paper takes the forest town as the research objects, and first explains the ecological welfare concept of the forest town. Secondly, the formation mechanism of ecological welfare is explored through the DPSIR conceptual model. Finally, constructing the ecological welfare evaluation index system of forest towns as Heilongjiang Province an example, and using the analytic hierarchy process to analyze the influencing factors. The research of this paper fills the blank of ecological welfare evaluation of forest towns, improves the theoretical basis of forest towns' ecological welfare, and provides a scientific basis for exploring the development path of forest towns' ecological welfare.

\section{The Concept of Ecological Welfare in Forest Towns}

Welfare is a synonym of well-being, which refers to all the beautiful living conditions or living environment of human beings. However, due to the late beginning of research on ecological welfare, the research has shown significant complexity, so the concept has not yet been able to form a unified understanding [11]. Zhang believes that ecological welfare is the result of the extension of welfare connotation and the popularization of ecological movement, and the extension of the concept of harmonious development between man and nature and society [12]. Tang believes that ecological welfare is an important indicator of human welfare [13]. Huang et al. believe that "ecological welfare" reflects the effect of the ecological environment on the level of human welfare and defines it as all material and nonmaterial, direct, and indirect benefits that a good ecological environment brings to people and the economy and society [14]. Zang et al. propose that ecological welfare is "the products and services that humans obtain or enjoy from the natural environment and are directly related to human wellbeing provided" [15]. Hu et al. considered that ecological welfare could divide into broad and narrow sense. In a narrow sense, ecological welfare refers to the direct or indirect benefits obtained from the natural ecosystem, and ecological welfare is an integral part of social welfare [16]. In a broad sense, ecological welfare is the comprehensive human welfare produced by human beings based on natural capital and combining human and material capital investment [17]. The economic and social development of forest towns is based on the advantages of abundant natural resources. At the same time, the natural ecosystem will also be affected by the economy and society. Therefore, forest towns can be regarded as a complex ecosystem composed of economy, society, and natural environment. Through field investigations in small towns, China's goal of building forest towns is organic to integrate "production, life, ecology, and culture." According to the goal of the construction, ecological welfare is not limited to the products and services provided by the natural ecosystem, it should be proposed from a broad perspective. Hence, the concept of ecological welfare is that forest towns' complex ecosystem provides the production function welfare, life function welfare, ecological function welfare, and cultural function welfare to the residents of the forest town through direct or indirect means (Figure 1).

\section{The Formation Mechanism of the Ecological Welfare of Forest Towns}

In 1993, the European Environment Agency (EEA) proposed the DPSIR model, which looks at the interaction between humans and environmental systems from the system analysis [18]. The DIPSR model comprises five dimensions: driving force, pressure, state, influence, and response. Among them, the development of society, economy, and population as the driving force (D) is the fundamental reason for the generation and development of the system. The driving force acts on the ecological environment, putting pressure on the natural ecological environment $(\mathrm{P})$, prompting changes in the state of the ecological environment (S), and then having various impacts on humans and the ecological environment (I). These influences prompt people to respond to changes in the environmental state (R). The response measures directly act on environmental pressure, state, and influence or act on the driving force constituted by the economy, society, and population. The ecological welfare of forest towns is also produced under the joint action of economy, society, and natural ecosystem. Hence, this paper adopts the DPSIR conceptual model to analyze the formation mechanism of ecological welfare.

In the forest town complex ecosystem, in order to promote economic and social development, increase the use of forest resources, and achieve ecological welfare, the driving force (D) is divided into three aspects: economic driving force, social driving force, and resource driving force. However, promoting urbanization in forest towns, the total population increases and the development and utilization of forest resources will inevitably consume ecological resources. And it has an impact on the ecological environment. So, the driving force causes pressure on the ecological environment (P). Driven by economic and social development, under the pressure of the destruction of the ecological environment, the affected ecological environment causes changes in the ecosystem in the forest town, disturbing the state of ecosystem services (S). Then, under the 


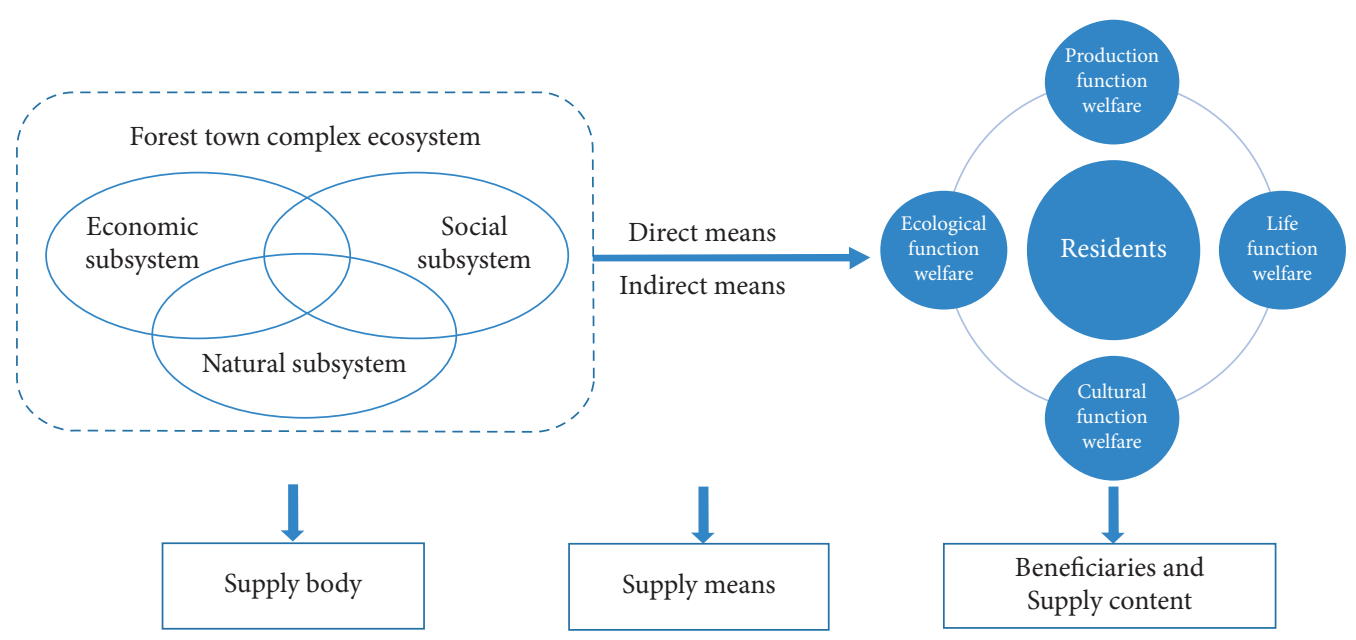

FIGURE 1: Ecological welfare of forest towns.

influence of the driving force (D), pressure (P), and state (S) of the forest town, the ecological welfare of the forest towns is formed, including eight aspects: income, medical care, employment, education, culture, social security, ecological environment, and infrastructure construction. Then, the town managers formulate policies and measures based on the current level of residents' ecological welfare and the states of the economic, social, and natural environment, which forms a response (R). The formulation of policies will promote the transition of economic and social development of production and lifestyle to green and low-carbon. By increasing financial investment in environmental and ecological governance, the pressure on the ecological environment will relieve, the condition of the ecosystem will improve and restore, and the level of ecosystem services will improve and then improve the ecological welfare of human beings. Meanwhile, response (R) will give feedback to ecological welfare (I) to verify whether the forest town development policy promotes the improvement of ecological welfare. The construction of the DPSIR model framework forms a multidimensional closed-loop circular structure of economy-society-nature-ecological welfare policy, which is helpful to analyze the formation process of ecological welfare (Figure 2).

\section{Construction of Ecological Welfare Evaluation Index System of Forest Towns}

4.1. Study Area-Forest Town in Heilongjiang Province. Heilongjiang Province is one of the largest forestry provinces in China. The total forestry management area of Heilongjiang is 31.75 million hectares, accounting for $2 / 3$ of the province's land area, and the forest coverage rate reaches $47.3 \%$. It is the most important state-owned forest area and the largest timber production base in China. There are more than 100 species of forest trees and more than 30 species of higher utilization value in Heilongjiang. In 2018, Heilongjiang occupies 8 of the 50 forest towns' construction pilot projects selected by the state. Abundant forest resources give Heilongjiang the advantage of building a forest town.
4.2. Research Method. The analytic hierarchy process (AHP) is a comprehensive evaluation method for system analysis and decision-making. It transforms human judgment into a comparison of the importance of several factors and more reasonably solves the shortcomings of decision maker's subjective judgments. This paper uses the analytic hierarchy process to calculate the weight of the influencing factors of the forest town's evaluation index so that we obtain the importance of the influencing factors. The calculation process is as follows:

(1) Establish a judgment matrix. Put the 16 influencing factors of the forest towns' sustainable development

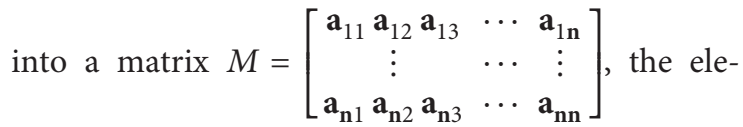
ments in the matrix correspond to $\mathbf{a}_{\mathbf{i j}}$ $(i, j=1,2, n) ; \mathbf{a}_{\mathbf{i j}}$ represents the importance of $i$ compared with index $j$, and $\mathbf{a}_{\mathbf{i j}}>0$ and $\mathbf{a}_{\mathbf{i j}} \times \mathbf{a}_{\mathbf{j i}}=1$, and it forms a positive and negative judgment matrix. Then, five experts in the group compare the indicators in the matrix pair by pair, score the importance of the two indicators, and fill in the average score in the matrix.

(2) Calculate the weight of the judgment matrix and check the consistency. Though using SPSSAU software, calculating the $\mathrm{CI}, \mathrm{CR}$, and weight values of the judgment matrix, conduct a consistency test. When $\mathrm{CR}<0.1$, the judgment matrix is considered to have satisfactory consistency, the weight of each indicator pass test.

4.3. Selection of Evaluation Index. The forest town is a functional community that integrates "suitable for production, livability, and travel," meeting people's survival and development needs by the forest town's "production, life, ecology, and culture." For the residents of forest towns, stable economic income, suitable jobs, a beautiful living environment, a healthy body, sound social security, perfect infrastructure construction, and a strong cultural 


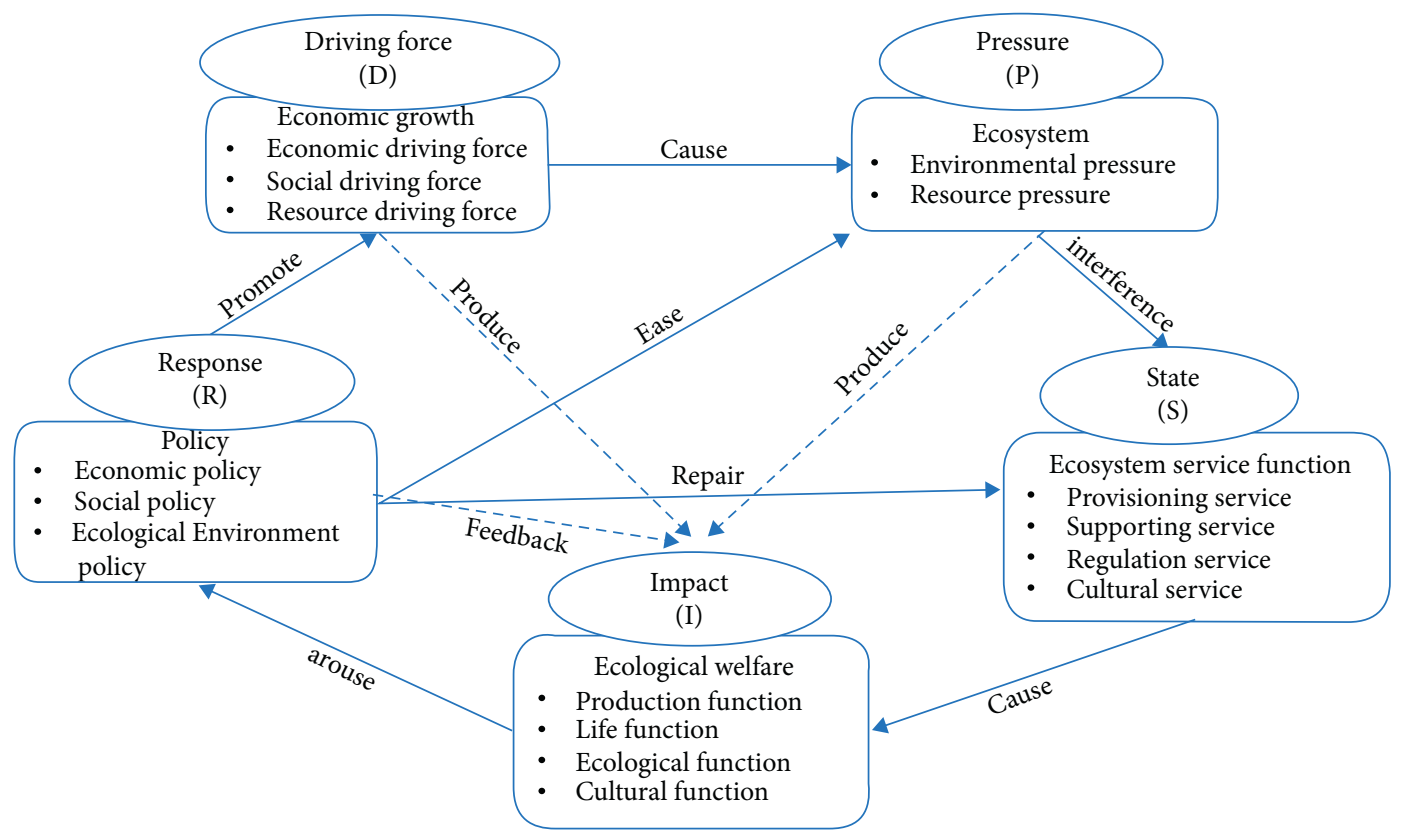

FIGURE 2: The formation mechanism of the ecological welfare based on the DPSIR conceptual model.

TABLE 1: Evaluation index system of forest town.

\begin{tabular}{|c|c|c|c|}
\hline Target level & Criterion level & Index level & Weights \\
\hline \multirow{2}{*}{ Production function } & Income level & Per capita GDP of forest town residents & $11.55 \%$ \\
\hline & Consumption level & Per capita consumption level of local residents & $7.27 \%$ \\
\hline \multirow{8}{*}{ Life function } & Medical service & Number of doctors per 10,000 people & $7.70 \%$ \\
\hline & Education level & Average years of education & $7.56 \%$ \\
\hline & \multirow{2}{*}{ Social security } & Basic medical participation rate & $5.87 \%$ \\
\hline & & Minimum living security rate & $3.98 \%$ \\
\hline & Employment level & Employment rate of residents in forest towns & $14.82 \%$ \\
\hline & \multirow{3}{*}{ Infrastructure services } & Road area per capita in the town & $2.65 \%$ \\
\hline & & Per capita housing area of forest town residents & $3.62 \%$ \\
\hline & & Internet penetration rate & $3.85 \%$ \\
\hline \multirow{4}{*}{ Ecological function } & \multirow{4}{*}{ Ecosystem environment } & Green coverage rate in built-up area & $3.55 \%$ \\
\hline & & Forest cover rate & $8.15 \%$ \\
\hline & & Good air quality rate & $8.15 \%$ \\
\hline & & Average negative oxygen ion concentration & $3.39 \%$ \\
\hline \multirow{2}{*}{ Cultural function } & \multirow{2}{*}{ Cultural service } & Number of heritages with forest culture and spiritual value & $3.96 \%$ \\
\hline & & Number of A-level scenic spots in forest town & $3.96 \%$ \\
\hline
\end{tabular}

atmosphere are their most cherished functions. Therefore, combined with the construction goals of forest towns, this paper will evaluate the ecological welfare of forest towns from the four aspects of the production function, life function, ecological function, and cultural function. By learning the welfare evaluation indicators from $\mathrm{Hu}$ et al. [16], Xiao Liming (2021) [19], Guo Lihua (2017) [20], Gao Bofa (2020) [21], and Ding Linlin (2017) [22], we selected the general indicators of the ecological welfare evaluation of forest towns. While choosing the characteristic indicators of the ecological welfare by referring An huben (2015) [23], Qin Yan et al. (2010) [24], Zhu Lin et al. [25], and Huang et al. [14]. The ecological welfare index system of forest towns with eight specifically functional activities are constructed, including income, consumption, medical care, education, social security, employment, infrastructure services, and cultural services (Table 1).

\section{Analysis of Evaluation Results}

It can be seen from Table 2 that the CR value of the judgment matrix is 0.039 , which is less than 0.1 , so the judgment matrix has a satisfactory consistency result. It can be concluded that, among the 16 indicators from the weight values in Table 1, the top four most important factors affecting the forest towns' ecological welfare are employment rate of residents, per capita GDP of forest town residents, forest cover rate, and good air quality rate. It shows that the production function is the most important and fundamental function for forest towns' residents. The employment welfare and income welfare brought by the production function are the foundation of survival for the residents of the forest towns. Besides that, an excellent ecological environment is also an essential factor influencing the ecological welfare of forest towns. The development of the town relies on abundant 
TABLE 2: The consistency test results.

\begin{tabular}{|c|c|c|c|c|}
\hline Largest characteristic root & CI value & RI value & CR value & The consistency test \\
\hline 16.94 & 0.063 & 1.594 & 0.039 & Pass \\
\hline
\end{tabular}

forest resources. The higher forest coverage rate is not only a guarantee for production but also an ecological barrier for residents' lives. However, the weight ratios of infrastructure services and cultural function welfare evaluation indicators in life functions are relatively small. This is because the residents of the forest towns primarily engage in forest tourism, forest product planting and breeding, or forest management and protection, and their overall income level is not high. Therefore, compared with living facilities and cultural atmosphere, they are more concerned about the economic benefits brought by forest resources.

\section{Conclusion}

Good ecological welfare can meet not only the increasing material and cultural needs of the people but also the ultimate goal of the development of forest towns. This paper puts forward the concept of forest town's ecological welfare based on the construction goal of forest town. Then, it explores the formation mechanism of ecological welfare through the DPSIR conceptual model. In addition, taking Heilongjiang Forest Town as an example, we construct four aspects of forest town ecological welfare evaluation index system including the production function welfare, life function welfare, ecological function welfare, and cultural function welfare. At last, through using the analytic hierarchy process to evaluate the importance of the indicators, the research of this paper provides a theoretical basis for the analysis of the ecological welfare development level of forest towns in the future. However, the collection of ecological welfare data of forest towns in this paper should be strengthened. Therefore, the data collection, the research on the improvement path of forest towns' ecological welfare and the interaction between ecological welfare and healthcare still need to be followed up.

\section{Data Availability}

The raw data supporting the conclusions of this article are available from the corresponding author upon request.

\section{Conflicts of Interest}

The authors declare that there are no conflicts of interest regarding the publication of this paper.

\section{Acknowledgments}

The work was supported by "The D-type project of the Fundamental Research Funds for the Central Universities" of "The model, PATH, and experimental demonstration of natural resources capitalization taking the forest ecological bank as an example" (2572020DZ09) and "New sustainable development based on the perspective of green finance to release resource advantages Mechanism Research" (2572020DY06).

\section{References}

[1] Millennium Ecosystem Assessment, Ecosystems and Human Well-Being: A Framework for Assessment, Island Press, Washing DC, 2003.

[2] S. Li, "How to scientifically measure the contribution of nature to mankind-A social-ecosystem analysis framework based on ecosystem services and its application," Academic Frontiers, vol. 11, pp. 28-35, 2020.

[3] S. Zhang, "Some thoughts on the development of forest towns," China Economic Report, vol. 3, pp. 95-98, 2017.

[4] Y. Ge, C. Cui, and Z. Chang, "Policy interpretation and path return to the healthy development of forest town-Taking Wugong forest town in Xianyang City as an example," Journal of Beijing Forestry University, vol. 3, pp. 49-54, 2020.

[5] J. Zhang, Y. Zeng, Y. Zhang, and B. Cheng, "Literature review and analysis of domestic forest town research," Forestry Economy, vol. 12, pp. 37-42+68, 2019.

[6] Y. Ge and J. Zhang, "Spatial distribution characteristics and influencing factors of forest characteristic towns in Zhejiang Province," Journal of Zhejiang AઐF University, vol. 2, pp. 374-381, 2020.

[7] B. Zhu, "Influencing factors and paths of the coordinated development of "big towns and small towns"-An empirical study based on the construction of characteristic towns in Zhejiang,” Academic Forum, vol. 1, pp. 116-121, 2018.

[8] C. Wang and S. Jia, "Characteristic index system and evaluation of Chinese characteristic towns," Nanjing Social Sciences, vol. 2, pp. 79-86+92, 2019.

[9] J. Tan and Y. Pan, "Performance evaluation of ecological welfare in characteristic towns-taking Chongqing as an example," Technology Management Research, vol. 24, pp. 40-46, 2019.

[10] T. Jiang, "Construction of the evaluation system for the tourism function of characteristic towns in Zhejiang Province," China's Agricultural Resources and Regionalization, vol. 6, pp. 227-232, 2019.

[11] Li Yan, S. Li, Y. Gao, and Y. Wang, "A framework for the classification of ecosystem services connecting multi-level human well-being," Acta Geographica Sinica, vol. 8, pp. 1038-1047, 2013.

[12] J. Zhang, "The rise of the concept of ecological welfare and the transformation of medical security model," Ecological Economy, vol. 1, pp. 90-92+116, 2009.

[13] J. Tang, "Three issues of ecological welfare-based on the perspective of ecological values," Journal of Nanchang University, vol. 4, pp. 12-20, 2020.

[14] Y. Huang, Z. Qiao, W. Zou, H. Qin, and X. Zhang, "Research on the evaluation index system of the ecological welfare supply system of national forest parks based on ISM," Acta Ecologica Sinica, vol. 10, pp. 4090-4098, 2021.

[15] Z. Zheng, D. Zheng, C. Sun, and X. Zou, "A multi-scale empirical test of the ecological curse effect in mainland China," Geographical Research, vol. 5, pp. 851-863, 2016. 
[16] M.-j. Hu, Z.-j. Li, Z.-s. Ding, N.-x. Zhou, D.-l. Qin, and C. Zhang, "Urban ecological well-being intensity and driving mode based on three-dimensional well-being:Taking the Yangtze Delta as an example," Journal of Natural Resources, vol. 36, no. 2, pp. 327-341, 2021.

[17] Z. Zheng and X. Zou, "Test of convergence characteristics of water resource intensity in mainland China: empirical evidence based on inter-provincial panel data," Journal of Natural Resources, vol. 6, pp. 920-935, 2016.

[18] European Environment Agency, Halting the Loss of Biodiversity by 2010: Proposal for a First Set of Indicators to Monitor Progress in Europe, EEA Copenhagen, Denmark, 2005.

[19] L. Xiao and Q. Xiao, "Analysis of the differentiation and spatial convergence of urban ecological welfare performance pattern in the Yellow River Basin," Soft Science, vol. 2, pp. 46-53, 2021.

[20] L. Guo and F. Wang, "Evaluation of changes in the welfare of ecological migration in pastoral areas from the perspective of feasible capabilities: taking Inner Mongolia and Qinghai as examples," Heilongjiang Ethnic Series, vol. 2, pp. 44-51, 2017.

[21] B. Gao, Li Cong, S. Li, and X. Han, "Research on the welfare status and influencing factors of relocated farmers in ecologically fragile areas," Resources and Environment in Arid Regions, vol. 8, pp. 88-95, 2020.

[22] L. Ding, Q. Wu, and Y. Li, "Research on the welfare changes of land-lost farmers under the background of new urbanization," China Population, Resources and Environment, vol. 3, pp. 163-169, 2017.

[23] H. An, Y. Fan, and B. Yang, "Research on sustainable development evaluation of forestry resource-based cities based on DPSIR model-taking Yichun as an example," Science and Technology Management Research, vol. 5, pp. 74-78, 2015.

[24] Y. Qin, S. Shen, and F. Wu, "The calculation method and application of the cultural function value of forest ecosystems: taking Zhangjiajie Forest Park as an example," Journal of Central South University of Forestry and Technology, vol. 4, pp. 26-30, 2010.

[25] L. Zhu, Li Lan, Z. Li, B. Fan, and D. Zhang, "Status and analysis of foreign forest cultural value evaluation indicators," World Forestry Research, vol. 5, pp. 92-96, 2015. 